\title{
Key to the Willows of Saskatchewan
}

\section{August J. Breitung}
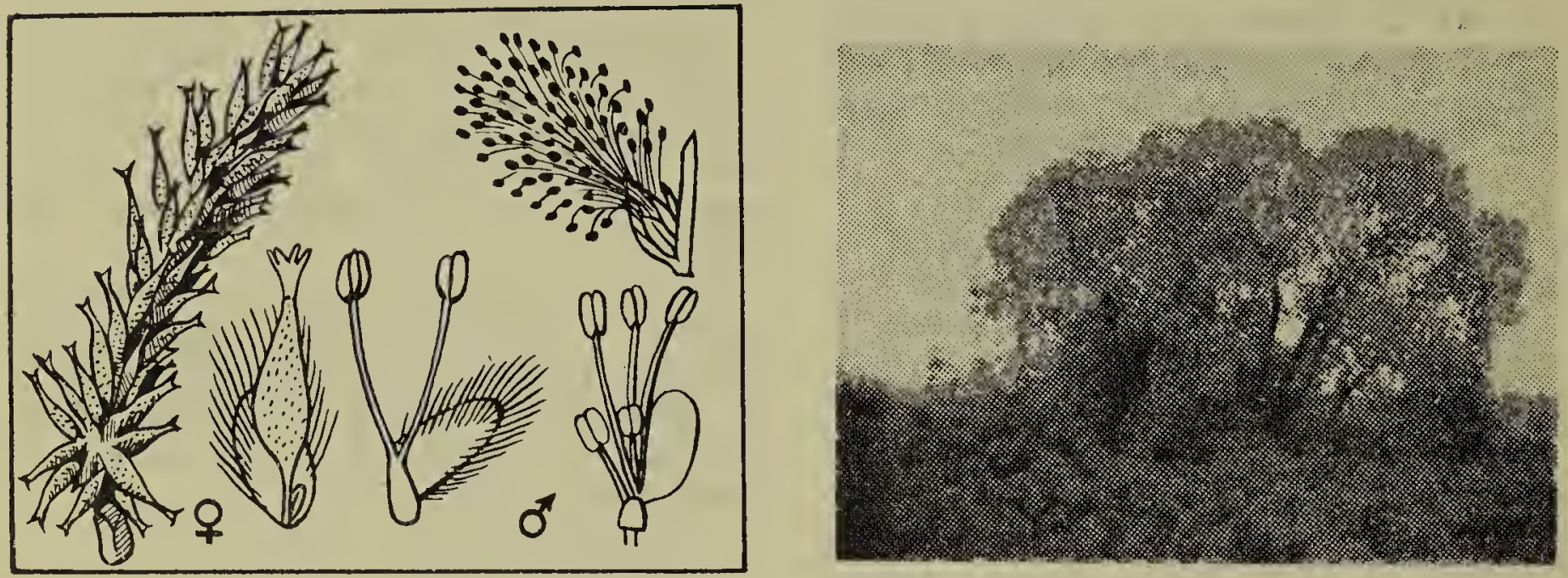

THERE are approximately 300 species of willows (Salix) widely distributed, mainly in the temperate and arctic regions of the northern hemisphere. Of this number at least 24 species are represented in Saskatchewan.

Following is a key, based largely on leaf characters, to all the willows known to occur in Saskatchewan south of the 54th parallel. Its purpose is to inspire extensive observation, stimulate collecting and thus increase our knowledge of the native flora. The key is intended to serve as a means to identify readily the willows of the inhabited regions and as a companion work to "Notes on the Willows of Saskatchewan" by the late Dr. W. P. Fraser in Can. Field-Nat. 56: 104-110, 1942. The willow taken to be Salix glauca var. glabrescens in the above publication has since been proven to be Athabasca willow (Salix athabascensis). The firm-leaf willow (Salix pseudocordata) and caudate willow ( $S$. caudata), both Cordilleran species, were found to occur in the Cypress Hills in 1947, and are additions to the Saskatchewan willow flora (See plates I and II).

\section{GENERIC CHARACTERS}

Shrubs or sometimes trees, with alternate leaves and single-scaled winter buds. Flowers dioecious (bearing stamens and pistils on separate plants), in sessile to long-peduncled catkins, appearing before or with the leaves; sepals and petals absent; stamens mostly 2-8 behind each scale-like bract; fruit a one-celled capsule with numerous, small, hair-tufted seeds.

\section{KEY TO SPECIES OF SALIX}

1. Leaves not hairy, or only slightly so when young.

2. Catkins appearing with the leaves; late flowering species.

3. Leaf-blades lanceolate to ovate, with ascending teeth; shrub not thicket-forming.

4. Stamens 3-8; bracts yellowish soon falling off.

5. Blades lanceolate to broadly lanceolate, acuminate, pale and glaucous beneath.

6. Leaves finely serrate, catkins loosely flowered; young $t$ wigs drooping ............................................................... S. amygdaloides. Peach-leaved W.

6. Leaves serrulate, catkins densely flowered, twigs not drooping S. lasiandra. Red W.

5. Blades narrowly lanceolate, long acuminate, green on both sides; Cypress Hills only ................................................................ S. caudata. Caudate W.

6. Leaf-blades ovate-lanceolate, abruptly long acuminate, green and shining on both sides; margin with 6-10 (12-15) teeth per $\mathrm{cm}$; flowering in May; capsules maturing in June S. lucida. Shining W. 
6. Leaves thick, shrub 5-10 feet high.

7. Leaf-blades ovate-lanceolate, or obovate oval, glandular serrulate, light green; stipules present; capsules glabrous; Cypress Hills only

S. pseudocordata. Firm-leaf W.

7. Leaf-blades finely serrulate, elliptic lanceolate or oblanceolate, dark green, stipules absent; capsules whitish hairy

S. MacCalliana. McCalla's W.

5. Blades glaucous beneath.

6. Shrub 5-10 feet high; leaves finely serrate, cordate at the base; female catkins 2-3 inches long; pedicels 6-8 $\mathrm{mm}$. long $S$. pyrifolia. Balsam W.

6. Shrub 2-3 feet high, leaves entire or nearly so, not cordate at the base, pedicels 3-5 $\mathrm{mm}$. long.

7. Leaves obovate or elliptic, thin; branchlets and capsules hairy; spruce swamps

S. athabascensis. Athabasca W.

7. Leaves oblong, obtuse, thick; branchlets and capsules not hairy;

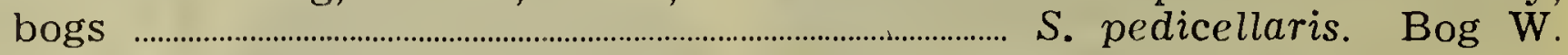

3. Leaf-blades linear with remote divergent teeth; stoloniferous, thicket forming shrub S. interior. Sandbar W.

2. Catkins appearing before the leaves; early flowering species.

3. Blades elliptic-lanceolate or ovate lanceolate to oval; rather coarsely wavy-toothed, stipules present, large, leafy

S. pseudomonticola. False-mountain W.

3. Blades lanceolate to elliptic; stipules absent, or when present, small.

4. Leaves lanceolate, shallowly toothed or entire, yellowish green; twigs yellow; old stems with diamond-shaped deformations, each resulting from a twig dying ........................... S. lutea. Yellow W.; Diamond W.

4. Leaves narrowly lanceolate, sharply toothed, dark green; twigs reddish, slender, stems without diamond-shaped scars S. petiolaris. Basket W.

5. Shrub or small tree 9-18 feet high; leaves lanceolate to obovate or elliptic, irregularly crenate-serrate, stipules present on vigorous sprouts, pedicels 3-4 mm. long ....................................................... S. discolor. Pussy W.

5. Shrub 6-9 feet high, branchlets maroon, leaves elliptic, pointed at both ends, entire or indistinctly toothed, stipules absent; capsules shortpedicelled ....................................................................... S. planifolia. Flat-leaved W.

1. Leaves hairy, even when mature.

2. Leaf blades hairy on both surfaces.

3. Lcaves, twigs and capsules grey-hairy, female catkins .5 inch long; shrub 1-2 feet high; alkaline meadows S. brachycarpa. Short-capsuled W.

3. Leaves, twigs and capsules white wooly; female catkins 1-2 inches long; shrub 3-5 (8) feet high; swamps and bogs .......... S. candida. Hoary W.

2. Leal-blades hairy beneath.

3. Blades ellintic, ovate to obovate.

4. Veins on the undersurface of the leaves prominent; margin sparcely serrale: (1) enlire, capsules long-pedicelled ........... S. Bebbiana. Bebb's W.

4. Veins on the undersurface of the leaves not prominent; capsules shortperlicelled.

5. Leaves oblanceolate to oblong, densely greyish, hairy beneath, wavy margined, shrub in sandy pine woods, Nipawin-castward

S. Scouleriana. Scouler's W.

5. Leaves ovate to obovate. silky beneath, entire; large shrub or small tree; Prince Albert-westward and C.ypress Hills ... S. Scouleriana. Scouler's W.

3. Blades lanceolate.

4. Lcaves lanceolate to linear-lanceolate, 3-6 inches long, entire, densely silvery-silky hencath; twigs often covered with a whitish bloom S. pellita. Satiny W.

4. Leaves narrowly lanceolate, 1.5-2 inches long, finely toothed, sparingly silveri-silky bencith; twigs maroon, without a bloom 